\title{
Medical Image of the Week: The Luftsichel Sign
}

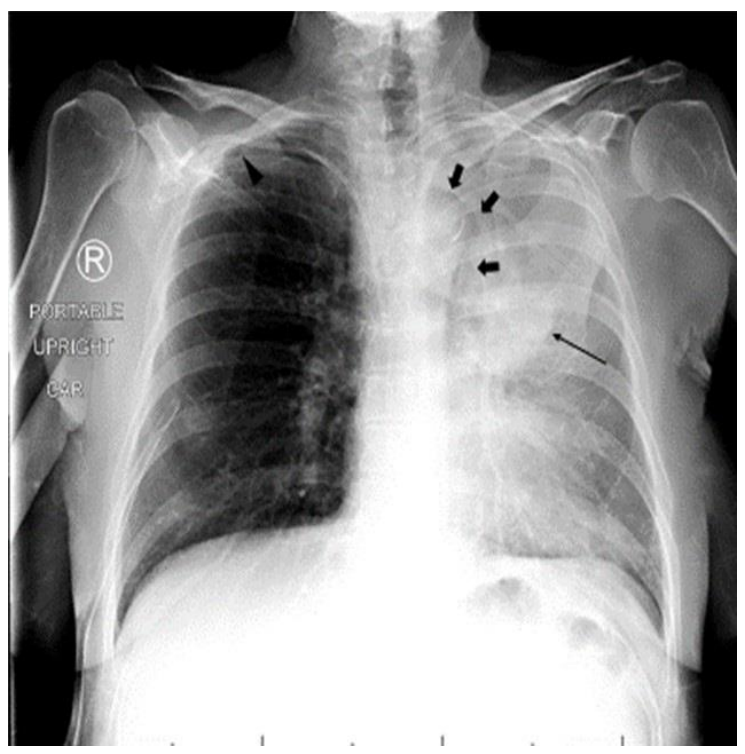

Figure 1. Anteroposterior chest radiograph demonstrating partial opacification of the left hemithorax, with preservation of the diaphragmatic border. A central mass is seen (thin arrow), as well as a radiolucent stripe bordering the aorta (thick arrows). Tracheal deviation to the left, a right-sided chest tube and a small right-sided pneumothorax are also noted.
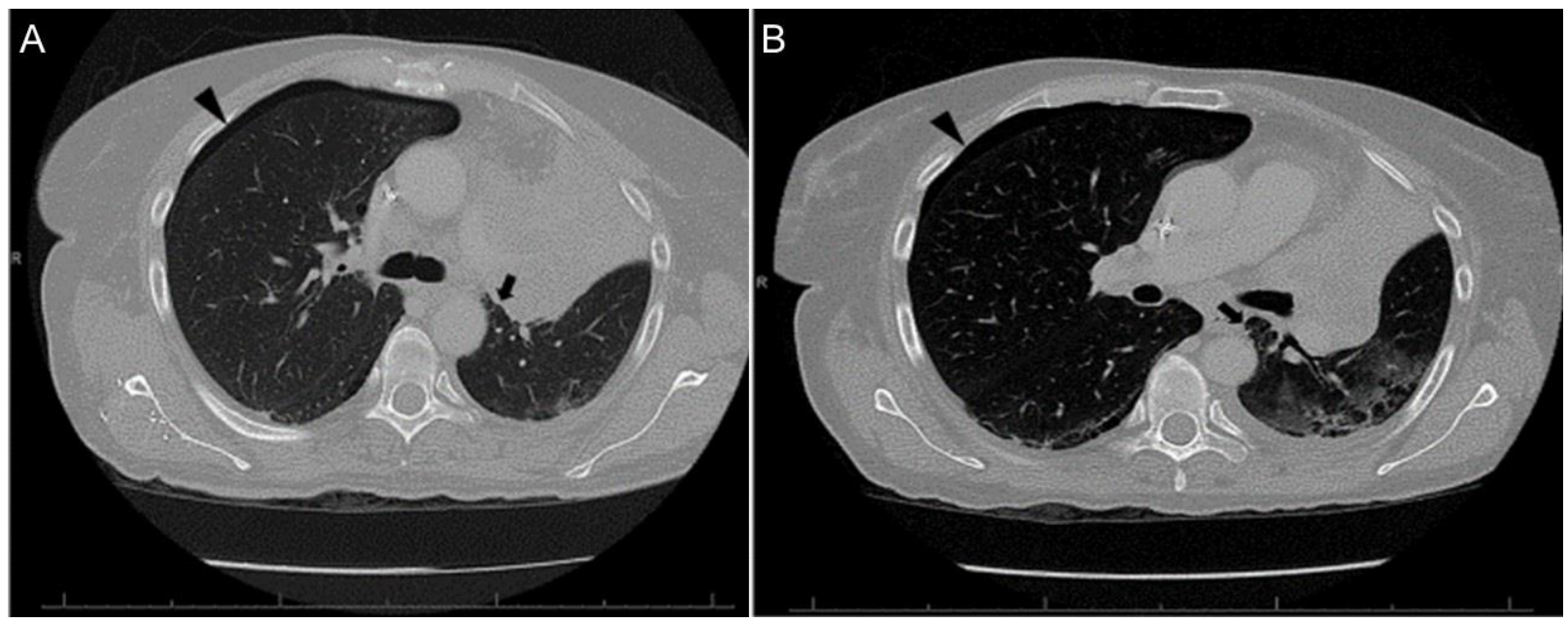

Figure 2. Axial computed tomographic of the chest at the level of the carina $(A)$ and left upper lobe bronchus (B) demonstrate opacification and volume loss of the left upper lobe with occlusion of the left upper lobe bronchus. The superior segment of the left lower lobe is interposed between the aorta and the atelectatic upper lobe (arrows). The right-sided pneumothorax is demonstrated and ground glass opacities are noted in the left lower lobe (arrowheads). 
A 59-year old woman with recently diagnosed small cell carcinoma with metastases to liver and spine presented after a fall presented with lower extremity weakness and incontinence. She was diagnosed with intertrochanteric femoral fracture and prior to planned transfer to our hospital for neurosurgical evaluation she underwent operative fixation of the fracture. An indwelling venous access port was also placed on the same day which was complicated by a pneumothorax requiring chest tube placement Upon arrival to our institution, she had normal vital signs and was in no distress. On respiratory examination, breath sounds were clear bilaterally on auscultation of the posterior chest but reduced on the left side on anterior auscultation. A chest tube was in place in the right mid-axillary line with no evidence of an air leak.

Chest x-ray demonstrated the right-sided chest tube and partial opacification of the left hemithorax, with a left hilar mass (Figure 1). The radiographic findings of left tracheal deviation, preservation of the left hemidiaphragm, and identification of the luftsichel sign suggested collapse of the left upper lobe. Computed tomography (CT) scan of the chest confirmed left upper lobar collapse due to extrinsic compression of the left upper lobar bronchus by a left upper lobe lung mass (Figure 2).

The luftsichel sign, a long-described marker of left upper lobe collapse on chest radiography, is a para-aortic stripe of radiolucency so-named for its course along the straight proximal descending aorta and curved aortic knob (in the German, luft for air, sichel for sickle) (1). Once theorized to be a result of herniation of the right lung into the left hemithorax after left-sided volume loss, CT correlation studies of radiographic signs in the 1980s verified the superior segment of the left lower lobe as the source of the lucency (2). Collapse of the left upper lobe displaces the major fissure anteriorly; the consequent movement of the left lower lobe results in expansion and interposition of its superior segment between the aorta and the atelectatic lung, as demonstrated in the correlate CT images in our patient.

Luke Gabe MD and Linda Snyder MD

Division of Pulmonary, Allergy, Critical Care and Sleep

Banner-University Medical Center, Tucson, AZ USA

\section{References}

1. Blankenbaker DG. The luftsichel sign. Radiology. 1998 Aug;208:319-20. [CrossRef] [PubMed]

2. Khoury MB, Godwin JD, Halvorsen RA Jr, Putman CE.CT of obstructive lobar collapse. Invest Radiol. 1985 Oct;20(7):708-16. [CrossRef] [PubMed] 